\title{
Medication errors in hospitals in the Middle East: a systematic review of prevalence, nature, severity and contributory factors
}

\author{
Binny Thomas ${ }^{1,2} \cdot$ Vibhu Paudyal $^{3} \cdot$ Katie MacLure $^{4} \cdot$ Abdulrouf Pallivalapila $^{1} \cdot$ James McLay $^{5} \cdot$ Wessam El Kassem $^{1}$. \\ Moza Al Hail ${ }^{1} \cdot$ Derek Stewart $^{6}$ (I)
}

Received: 8 December 2018 / Accepted: 2 May 2019 / Published online: 24 May 2019

(C) The Author(s) 2019

\begin{abstract}
Purpose The aim was to critically appraise, synthesise and present the evidence of medication errors amongst hospitalised patients in Middle Eastern countries, specifically prevalence, nature, severity and contributory factors.

Methods CINAHL, Embase, Medline, Pubmed and Science Direct were searched for studies published in English from 2000 to March 2018, with no exclusions. Study selection, quality assessment (using adapted STROBE checklists) and data extraction were conducted independently by two reviewers. A narrative approach to data synthesis was adopted; data related to error causation were synthesised according to Reason's Accident Causation model.

Results Searching yielded 452 articles, which were reduced to 50 following removal of duplicates and screening of titles, abstracts and full-papers. Studies were largely from Iran, Saudi Arabia, Egypt and Jordan. Thirty-two studies quantified errors; definitions of 'medication error' were inconsistent as were approaches to data collection, severity assessment, outcome measures and analysis. Of 13 studies reporting medication errors per 'total number of medication orders'/ 'number of prescriptions', the median across all studies was 10\% (IQR 2-35). Twenty-four studies reported contributory factors leading to errors. Synthesis according to Reason's model identified the most common being active failures, largely slips (10 studies); lapses (9) and mistakes (12); error-provoking conditions, particularly lack of knowledge (13) and insufficient staffing levels (13) and latent conditions, commonly heavy workload (9).

Conclusion There is a need to improve the quality and reporting of studies from Middle Eastern countries. A standardised approach to quantifying medication errors' prevalence, severity, outcomes and contributory factors is warranted.
\end{abstract}

Keywords Medication errors $\cdot$ Prescribing errors $\cdot$ Error causation $\cdot$ Systematic review $\cdot$ Middle East

Electronic supplementary material The online version of this article (https://doi.org/10.1007/s00228-019-02689-y) contains supplementary material, which is available to authorized users.

Derek Stewart

d.stewart@rgu.ac.uk

1 Medication Safety and Quality Center, Pharmacy Executive Office, Hamad Medical Corporation, PO Box 3050, Doha, Qatar

2 School of Pharmacy and Life Sciences, Robert Gordon University, Aberdeen AB10 7JG, UK

3 School of Pharmacy, University of Birmingham, Birmingham B15 2TT, UK

4 School of Pharmacy and Life Sciences, Robert Gordon University, Aberdeen AB10 7JG, UK

5 The Institute of Applied Health Sciences, University of Aberdeen, Aberdeen AB25 2ZD, UK

6 College of Pharmacy, Qatar University, Doha PO Box 2713, Qatar

\section{Introduction}

In 1999, the 'Institute of Medicine' (now the National Academy of Medicine) published the seminal report 'To Err Is Human: Building a Safer Health System' quantifying the scale of harm associated with medical care in the United States (US) [1]. The authors called for coordinated efforts by governments, healthcare providers and consumers and others to promote patient safety, setting a minimum goal of 50\% reduction in medical errors by 2004. Despite global advances in healthcare practices, an estimated one in ten patients is still harmed while receiving care [2]. In March 2017, the World Health Organization (WHO) published 'Medication Without Harm, WHO Global Patient Safety Challenge' [3, 4]. It called for action to reduce patient harm which occurs as a result of unsafe medication practices and medication errors. The aim is to 'gain worldwide commitment and action to reduce severe, avoidable medication-related harm by $50 \%$ in the next 5 years, 
specifically by addressing harm resulting from medication errors or unsafe practices due to weaknesses in healthcare systems'. One key objective is to 'assess the scope and nature of avoidable harm and strengthen the monitoring systems to detect and track this harm' $[3,4]$.

A number of published systematic reviews have attempted to quantify medication errors at various stages of the medication use processes of prescribing, transcribing, verifying, administration, dispensing and monitoring [5-21]. These have largely focused on secondary care inpatients, with most reporting errors committed in targeted groups of patients including paediatrics, acute care, older people, mental health and perioperative care. Many of these reviews also reported data on contributory factors leading to errors $[6,9-11,14,17$, 21]. One key limitation highlighted in many of these reviews is the lack of a standardised approach to defining and measuring errors, limiting the validity of any pooling of data from different studies and different systematic reviews. Furthermore, the very different healthcare structures and processes across the world may limit the generalisability of findings to other contexts. Given the first objective of the WHO challenge, there may be merit in conducting systematic reviews capturing studies from specific contexts to provide the most meaningful data which can be used to inform future strategies and interventions.

Given the differing healthcare systems, ethnicity, culture and work practices of the Middle East, there may be merit in conducting systematic reviews of studies within that geographical area (i.e. Bahrain, Egypt, Iran, Iraq, Israel, Jordan, Kuwait, Lebanon, Oman, Palestine, Qatar, Saudi Arabia, Syria, Turkey, United Arab Emirates and Yemen). In 2013, Alsulami et al. published a systematic review of studies up to and including 2011 on the incidence and types of medication errors in Middle Eastern countries and main contributory factors [10]. While noting that error rates were difficult to compare between studies due to being expressed differently, prescribing errors ranged from $7.1 \%$ of prescriptions in a teaching hospital to $90.5 \%$ of prescriptions in a primary healthcare centre. Poor knowledge of medicines was identified as a contributory factor for errors by doctors and nurses.

One limitation of this review was the lack of any theories of error causation in the synthesis stage. Incorporation of theory in primary studies or systematic reviews will yield findings which provide more comprehensive coverage of the key influential factors. The most commonly used and cited theoretical framework in this field is Reason's Accident Causation model. This model groups error causes as follows:

1. Active failures which are unsafe acts committed by people who are in direct contact with the patient or system. They take a variety of forms including slips and lapses (errors in task execution), mistakes (errors in planning) and procedural violations (rule breaking).
2. Error-producing conditions which can have adverse effects of error-provoking conditions within the local workplace (e.g. time pressure, understaffing, inadequate equipment, fatigue and inexperience).

3. Latent failures which arise from decisions made by policy makers, leaders and top-level management [22].

Furthermore, the review highlighted that published papers from Middle Eastern countries were relatively few and generally of poor quality. Given the advances in healthcare in recent years, an updated systematic review incorporating error theory is warranted.

The aim of this systematic review was to critically appraise, synthesise and present the available evidence of medication errors amongst hospitalised patients in Middle Eastern countries, specifically prevalence, nature, severity and contributory factors.

\section{Methods}

The systematic review protocol was developed in accordance with the Preferred Reporting Items for Systematic Review and Meta-Analysis Protocols (PRISMA-P) guidelines [23] and registered with the International Prospective Register of Systematic Reviews (PROSPERO, CRD42015019693) [24].

\section{Inclusion and exclusion criteria}

Primary research studies of any design conducted in hospital settings in the Middle East (as defined in the introduction) which quantified medication errors (i.e. prescribing, administration or dispensing errors) published as full papers in English from 2000 to the end of March 2018 were included in the review. Studies which reported error nature, severity or associated causative factors were also included. Studies of adverse drug events which were not classified as errors were excluded, as were review articles, letters, opinion papers, editorials and conference abstracts.

\section{Search strategy}

The search was conducted in Cumulative Index of Nursing and Cumulative Allied Health Literature (CINAHL), Embase, Medline, Pubmed and Science Direct. Search terms (title, abstract, text, keyword) were (medic* OR prescrib* OR dispens* OR administ*) AND (error* OR incident* OR mistake*) AND (Middle East OR Bahrain OR Egypt OR Iran OR Iraq OR Israel OR Jordan OR Kuwait OR Lebanon OR Oman OR Palestine OR Qatar OR Saudi Arabia OR Syria OR Turkey OR United Arab Emirates OR Yemen). The reference lists of all identified papers were reviewed to identify additional studies. 


\section{Screening}

Screening of titles (BT, DS), abstracts (BT, DS) and full papers (BT, DS) was independently performed by two reviewers, with disagreements resolved by consensus and referred to a third reviewer (KM) whenever required.

\section{Assessment of methodological quality}

Papers were independently assessed for methodological quality by two reviewers (BT and one of DS, VP, AP, JM, WEK, $\mathrm{MAH}$ ) with disagreements resolved by consensus and referred to a third reviewer whenever required. The STROBE checklist (STrengthening the Reporting of OBservational studies in Epidemiology) was adapted as a quality assessment tool [25]. For all study designs, STROBE criteria retained were those relating to bias with addition of criteria specific to medication errors (e.g. error definitions). For qualitative studies, credibility and dependability replaced validity and reliability, and transferability replaced generalisability.

\section{Data extraction}

A bespoke data extraction tool was developed and piloted to extract the following: authors, country of publication/study, year of publication, study population, setting, recruitment, error quantification, nature of errors, error severity and contributory factors. Data extraction was also performed by two independent reviewers, as per quality assessment.

\section{Data synthesis}

Previous systematic reviews have highlighted the heterogeneity of studies in terms of error definitions, methods of measurement and outcome measures [5-21]; hence, a narrative approach to data synthesis was selected a-priori. Data related to error causation were synthesised using Reason's Accident Causation model as a theoretical framework in terms of active failures, error-producing conditions and latent failures [22].

\section{Results}

\section{Study screening}

Database searching and review of reference lists yielded 452 articles, 110 of which were duplicates and excluded. Review of titles and abstracts excluded 213 papers with full-paper review excluding a further 79. Fifty papers were included in the quality assessment stage. The PRIMSA flowchart is given in Fig. 1. Of the fifty studies, 48 were of a quantitative, crosssectional design and two were qualitative in nature.

\section{Quality assessment}

Of the 50 studies, none met all 11 STROBE-related quality assessment criteria. Thirteen studies (26\%) met eight or more criteria, $21(42 \%)$ between five and seven criteria and the remaining $16(32 \%)$ meeting four or less. Key limitations centred on lack of justification for the method of sampling and sample size, and not adequately considering issues of data validity and reliability (quantitative studies) and trustworthiness (qualitative studies). Supplementary Table 1 gives the findings of the quality assessment processes.

\section{Characteristics of included studies}

Almost half of the studies were conducted in Iran $(n=23$, $46 \%$ ), followed by Saudi Arabia $(n=10,20 \%)$, Egypt and Jordan $(n=5$ each, $10 \%)$, Turkey $(n=2,4 \%)$ and one each (2\%) from Israel, Qatar, Yemen, Palestine and Lebanon. None of the studies reported data from more than one country. Two thirds $(n=33,66 \%)$ were conducted in university-affiliated or academic hospitals, one fifth $(n=10,20 \%)$ tertiary care nonteaching hospitals and only three $(6 \%)$ in general hospitals. Three studies $(6 \%)$ did not state the type of hospital and one $(2 \%)$ reported an analysis of a national online database. Within each hospital, a range of specific patient groups was targeted, mostly adults, and the most common types of wards chosen were intensive care units.

The definition of medication errors (or sub-categories of medication errors) was inconsistent. In the 50 studies, 17 different definitions were given, differing in wording and content. The most widely used was that of the US National Coordinating Council for Medication Error Reporting and Prevention (NCCMERP) [26]. Ten studies (20\%) adopted non-standardised definitions from previous studies or provided their own definition. Three studies (6\%) used the definition of medication errors as per Aronson et al. [27]. Two studies (4\%) on prescribing errors used the definition of the American Society of Health-System Pharmacists [28]. One study each used definitions provided by Dean et al. [29] and the Institute of Medicine [30]. Twelve studies (24\%) did not provide any definition of either medication errors or the sub-category being reported.

\section{Quantifying medication errors}

Of the 32 studies quantifying medication errors, the most common methods of data collection were via review of medication charts or records (prescribing, dispensing and administration) $(n=11,31 \%)$ or by analysis of data from an error or incident monitoring system $(n=9,28 \%)$. Only one study employed multiple approaches to data collection. Data collection periods ranged from 20 days to 2 years. Data extraction of the 32 studies is provided in Supplementary Table 2. 
Fig. 1 PRISMA flowchart describing systematic review search and study selection

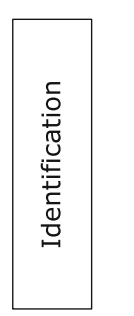
Records identified through
database searching $(n=445)$

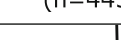

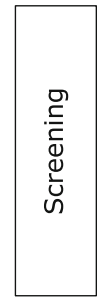
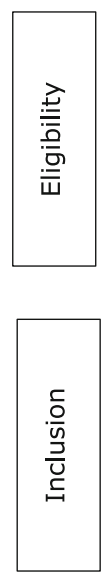
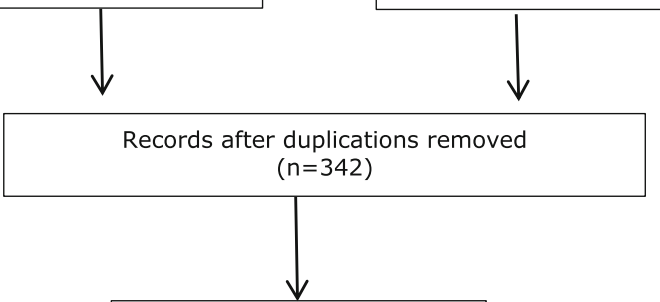

Titles and abstract screened $(n=342)$
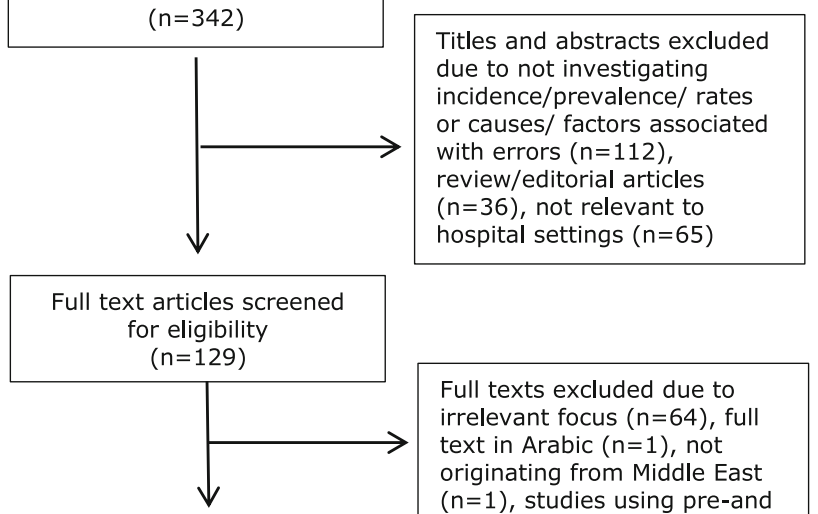

Full texts excluded due to irrelevant focus $(n=64)$, full

text in Arabic $(n=1)$, not originating from Middle East $(n=1)$, studies using pre-and post-interventional methods $(n=13)$

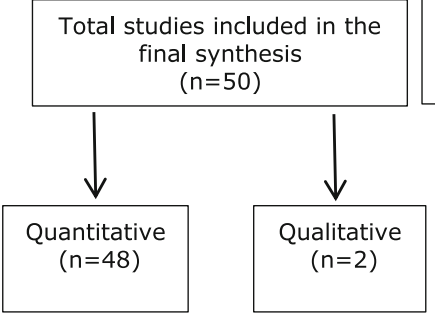

Inconsistencies in definitions of 'medication error', 'prescribing error' etc., together with the vast range of approaches to data collection and presentation of findings, limited pooling of data hence a narrative approach to data synthesis was employed. Almost half of the studies $(n=32,47 \%)$ quantified 'medication errors' in general, with fewer solely reporting 'administration errors' ( $n=7,22 \%)$ or 'prescribing errors' $(n=6,18 \%)$ and one $(3 \%)$ reporting only transcribing errors. Three studies reported data with combinations of classifications of medication errors.

The specific terms used in the studies to report medications errors varied and eight different denominators were used, the most frequent being 'total number of medication orders' or 'number of prescriptions' ( $n=13,40 \%)$, followed by 'number of patients admitted' ( $n=6,19 \%)$ and 'total number of opportunities for errors' $(n=4,12 \%)$. One study $(3 \%)$ each used 'total number of preparations', 'total number of medications dispensed', 'total number of cases/records', 'total number of patient days' and 'total number of reports'. Four studies (13\%) did not specify the denominator.
Given this marked heterogeneity, it was not possible to make valid comparisons of the outcome measure of prevalence. Even in studies which used the same outcome measure, the error definitions and methods of measurement varied considerably. The following results should therefore be interpreted with caution.

Of the 13 studies reporting medication errors per 'total number of medication orders'/'number of prescriptions', the median across all studies was $10 \%$ (IQR 2-35\%). The rates varied from 0.18 to 56 per 100 medication orders'/'number of prescriptions'. Of the six studies reporting 'number of patients admitted', the median was $28 \%$ (IQR 1-35\%), varying from 0.15 to 40 errors per 100 patient admissions.

\section{Nature and severity of medication errors}

Almost all studies $(31 / 32,97 \%)$ provided data regarding the nature of the errors. For prescribing errors, the most commonly reported included errors of omission, wrong drug, wrong dose, wrong route, incomplete order, wrong duration, drug- 
drug interaction and wrong patient. Studies reporting administration errors were largely related to wrong administration time, wrong administration route and wrong infusion rate.

Fourteen studies $(43 \%)$ reported the specific medications most commonly associated with errors. Most frequently reported therapeutic groups included anti-infectives for systemic use, drugs used for alimentary tract and metabolism and cardiovascular drugs.

Thirteen studies (40\%) reported error severity, with eight categorising according to the NCCMERP Index [26]. These studies, however, provided very little methodological detail on the application of the index, specifically assessment of interrater reliability. In five studies, the most common category was $\mathrm{B}$ (near miss), with $\mathrm{C}$ (error occurred and reached the patient but with no harm) in two studies and $\mathrm{E}$ (error occurred and may have contributed to or resulted in temporary harm and required intervention) in one study.

\section{Contributory factors}

Twenty-four studies (48\%) from six Middle-Eastern countries reported causes or contributory factors leading to medication errors. Approaches to data collection were largely based on questionnaires $(15 / 24,63 \%)$, data from incident reporting systems $(n=4,17 \%)$, direct observation of practice $(n=2,8 \%)$, semi-structured interviews $(n=2,8 \%)$ and retrieval of information from patient medical records $(n=1,4 \%)$. A total of 3919 health professionals were involved in these 24 different studies. Notably, none of these 24 studies used any theory (e.g. behavioural, organisational) in the processes of data collection or analysis. As described in the methods section, findings from these 24 studies were categorised according to Reason's Accident Causation model [22] (Table 1), and synthesis of the categories is provided in Table 2. Contributory factors most commonly reported were active failures, largely slips, lapses and mistakes; error-provoking conditions, particularly those relating to lack of knowledge and insufficient staffing levels and latent conditions, most commonly heavy workload. Error-provoking conditions such as lack of experience, poor documentation and look-alike drugs, or latent conditions of issues relating to a blame culture were rarely reported.

\section{Discussion}

\section{Statement of key findings}

Heterogeneity in medication error definitions and scope, differences in methods of data collection and units of analysis of the studies included in this review limited data pooling. Most frequently reported was the percentage of medication errors per total number of medication orders with a median across all studies of $10 \%$ (IQR 2-35\%). Prescribing errors were the most common type of errors reported, with dose-related errors being most prevalent. Contributory factors associated with medication errors were multifactorial. Synthesis of findings according to Reason's Accident Causation model identified that active failures (slips, lapses and mistakes) were most commonly reported followed by error-provoking conditions (e.g. lack of knowledge, insufficient staffing), with latent failures (e.g. heavy workload) least reported.

\section{Strengths and weaknesses}

There are several strengths to this review. The protocol was developed according to the standards of PRISMA-P (Preferred Reporting Items for Systematic review and MetaAnalysis Protocols [23], registered in the PROSPERO database [24], and the systematic review reported according to PRISMA (Preferred Reporting Items for Systematic Review and Meta-Analysis) criteria [55]. The synthesis adopted a theory-driven approach based on Reason's Accident Causation Model [22], which could subsequently facilitate the development of interventions. There are, however, several weaknesses; hence, the review findings should be interpreted with caution. Restricting the search to the English language and excluding those written in regional languages of Arabic or Persian may have limited retrieval of potentially relevant studies. It is, however, worth noting that English is the preferred language of most professional organisations in the Middle East.

\section{Interpretation of key findings}

Although there has been an increase in the number of medication errors studies originating from Middle East over the last few years, two thirds were from Iran and Saudi Arabia with none from eight countries. While the reasons for the lack of studies in other countries are unknown, this does have implications for the generalisability and transferability of review findings and conclusions. Furthermore, there was a lack of studies employing a qualitative approach to explore contributory factors of errors.

The majority of studies had key limitations in study design and lacked transparency in reporting key study details. Authors should be encouraged to adopt standardised reporting checklists available from the EQUATOR (Enhancing the QUAlity and Transparency Of health Research) network [56]. This international network aims to 'improve the reliability and value of published health research literature by promoting transparent and accurate reporting.' An example is the STROBE checklist (Strengthening the Reporting of Observational Studies in Epidemiology) for reporting observational studies [25]. 


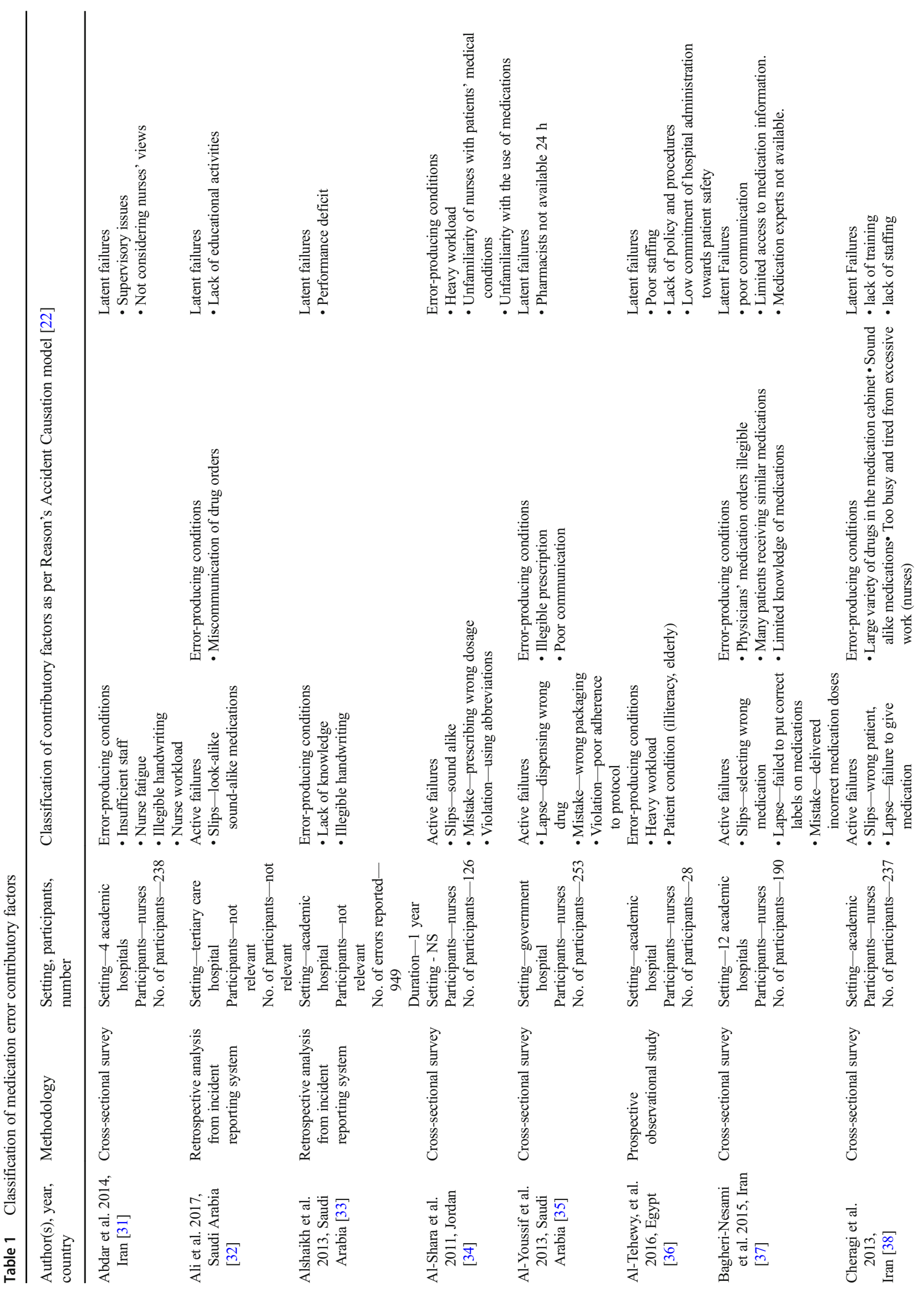




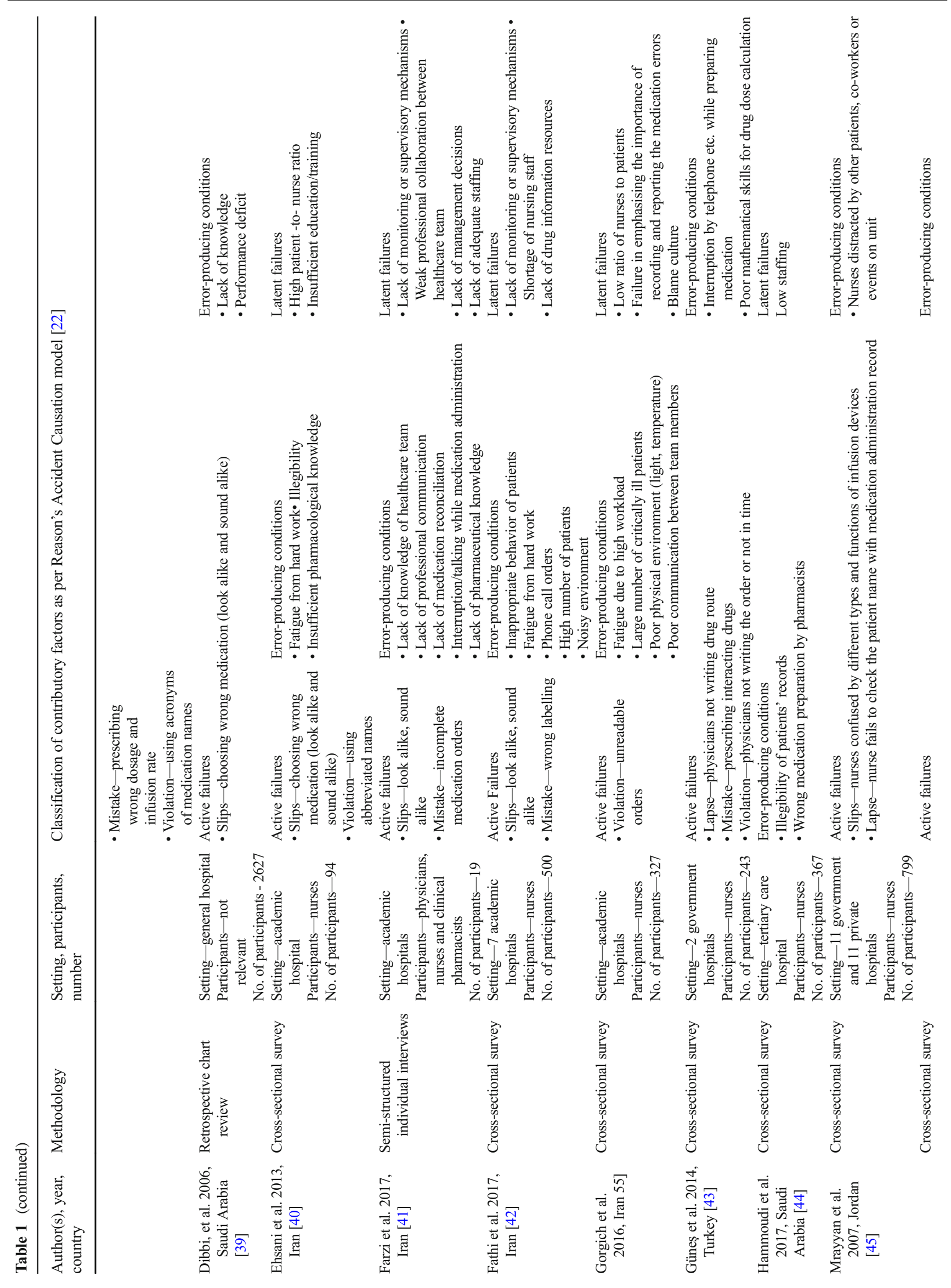



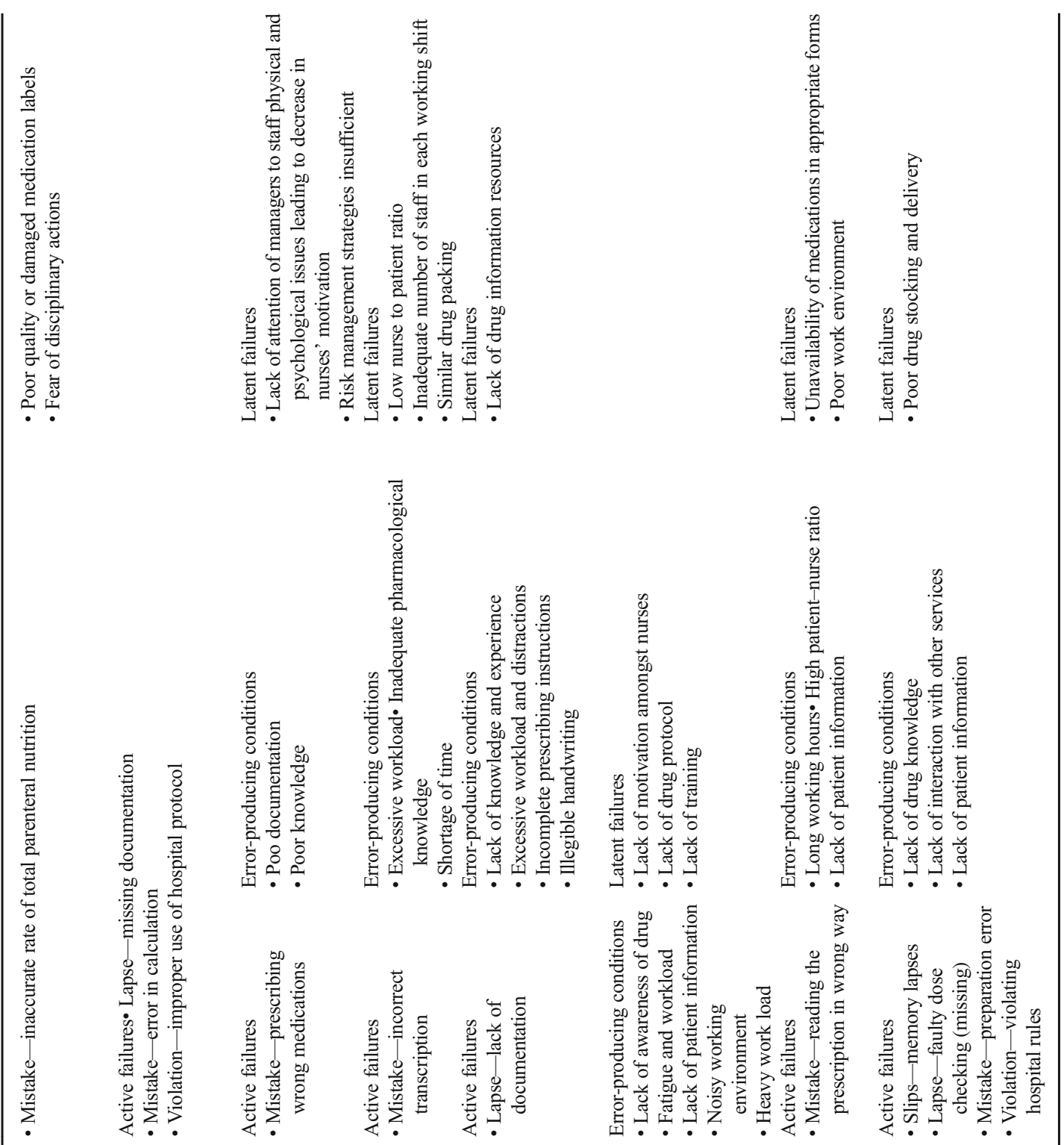

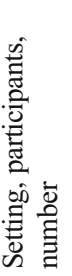

चे

茾高

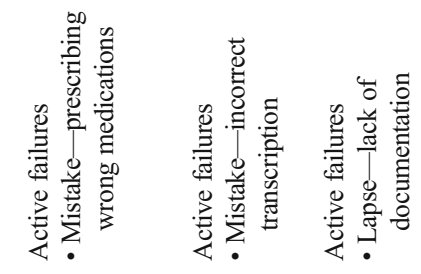

,

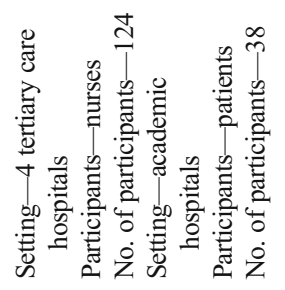

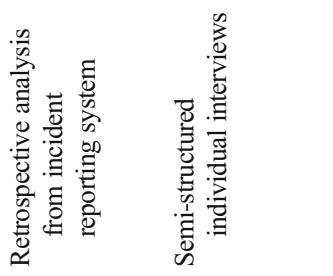

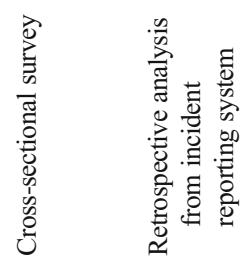

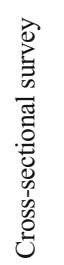

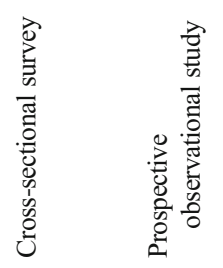

(1)

望毫

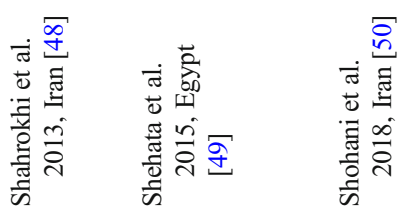

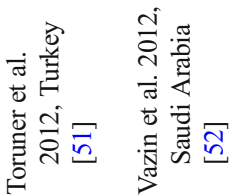




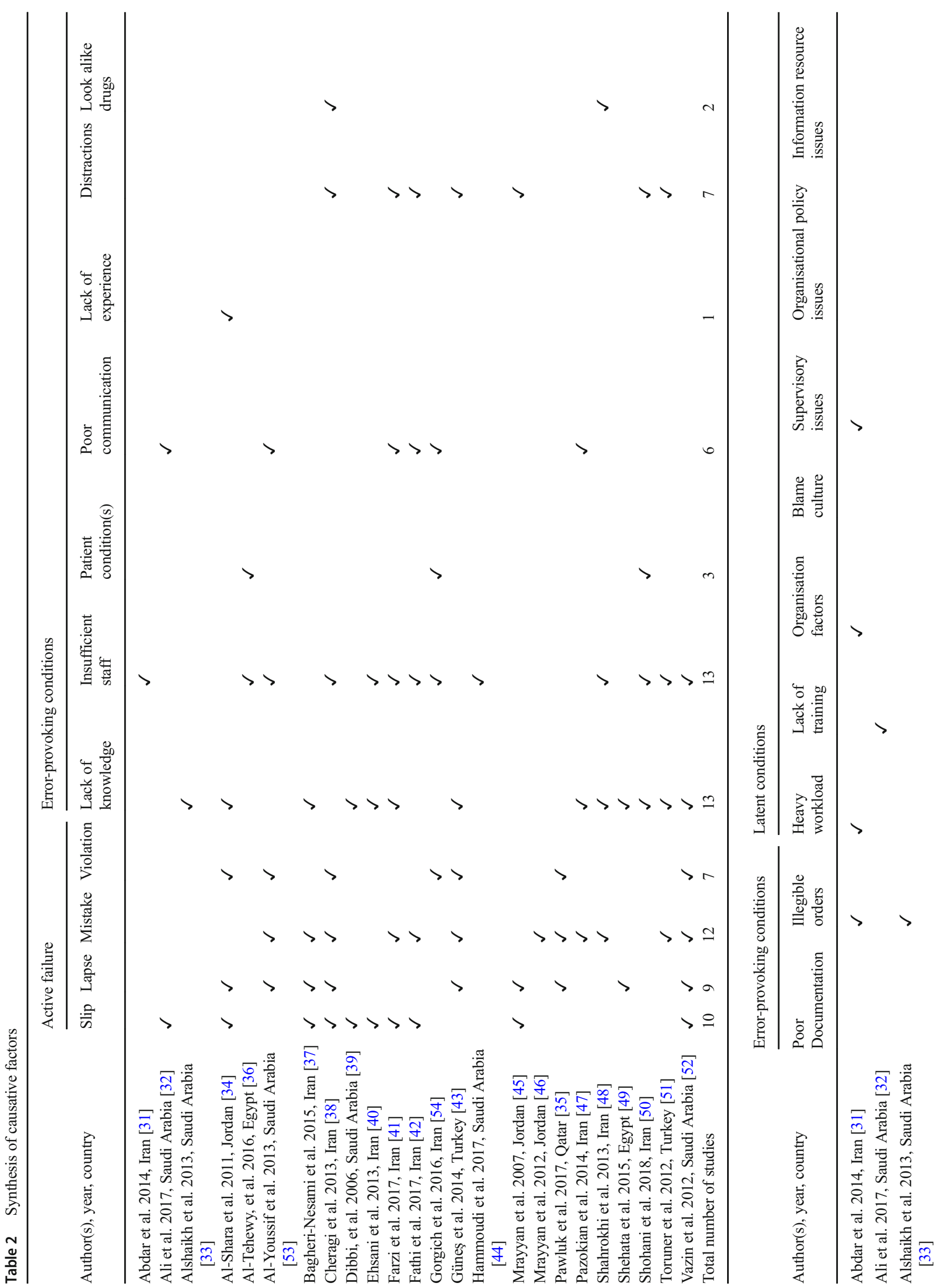


1278

Eur J Clin Pharmacol (2019) 75:1269-1282

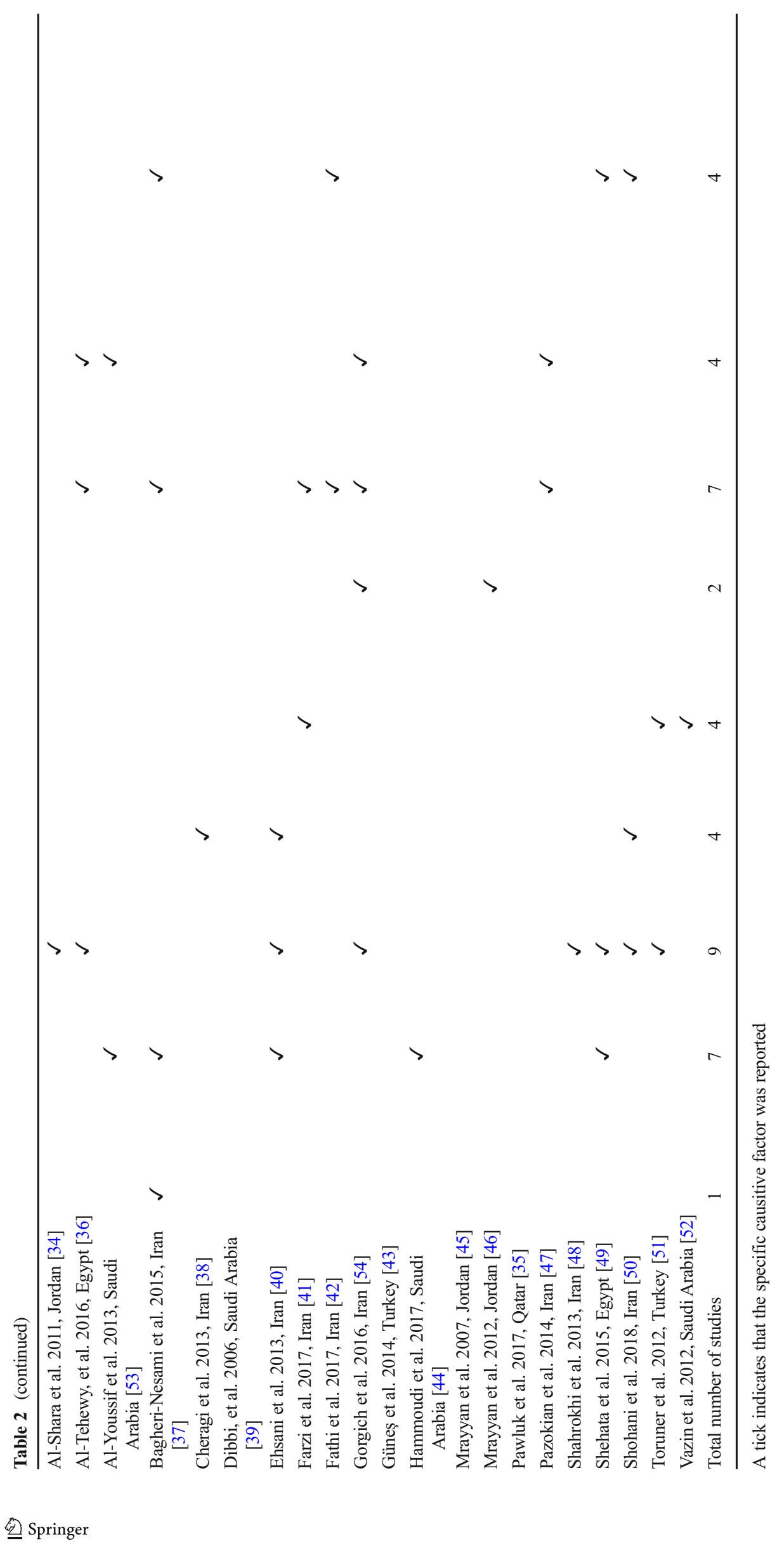


As noted in previous systematic reviews [5-21], many studies either did not define terms such as 'medication errors', 'prescribing errors' etc. or used non-standardised definitions. There was also variation in the methods used and the duration of data collection. To further advance this field of research, the adoption of standardised definitions and methodologies should be encouraged. This would enable analytical approaches such as meta-analyses and provide more robust and generalisable findings to inform practice.

Few studies reported the severity of errors, often providing little methodological detail. In a systematic review of tools used in error severity estimation, Garfield et al. highlighted that of the 40 tools assessed, only two were deemed to have acceptable validity and reliability [57].

Despite these issues around standardisation, it is evident from this systematic review that medication errors remain prevalent in hospitals in the Middle East. For those reporting medication errors, the median 'total number of medication orders'/ 'number of prescriptions' across all studies was $10 \%$ (IQR 2-35\% and range of $0.18-56 \%$ ). While differences in methodology, settings and patient populations limit comparisons to other systematic reviews; these figures are similar to those reported by Alsulami et al. in a systematic review of Middle Eastern studies up to 2011 [10]. The prevalence of medication errors in the Middle East would appear to remain largely unchanged and at a similar level to those reported from around the world [5-21].

None of the 24 studies in this review and only two previous systematic reviews analysed causative factors according to Reason's theory. In a review of prescribing errors in hospitalised patients, Tully et al. reported that the active failure most frequently cited was a mistake due to inadequate knowledge of the drug or the patient. There were issues of lack of training or experience, fatigue, stress, high workload and inadequate communication between healthcare professionals [9]. In a systematic review of medication administration error studies, Keers et al. reported that slips and lapses were the most common unsafe acts [11]. Our synthesis of study findings according to Reason's Theory is similar in that active failures of slips, lapses and mistakes were most common. Error-provoking conditions included lack of knowledge and insufficient staff. It is possible that other contributory factors may have been identified if the primary studies had used Reason's Theory in data collection and analysis. Using a theoretical framework in primary research would ensure that all possible explanations underlying medication errors are identified [58]. Given the accumulation of evidence from this and other systematic reviews, a standardised, theory-informed approach should be adopted. This is fundamental to the key stated WHO objective of assessing and scoping the nature of avoidable medication-related harm [3, 4].

Policy makers, leaders, practitioners and other relevant stakeholders must continue working towards minimising the key-identified contributory factors where possible.

\section{Further research}

There is a need for consensus-based research to define and standardise medication error definitions, approaches to data collection and outcome measures. Furthermore, theoretically informed qualitative research which allows in-depth exploration of contributory factors leading to medication errors is warranted. The findings from studies such as these would facilitate the development, testing, evaluation and monitoring of interventions aiming to reduce avoidable medication-related harm. There is evidence that consideration of theory allows comprehensive identification of the key issues to be targeted as part of intervention development leading to more effective and sustainable interventions compared to more pragmatic approaches [58].

\section{Conclusion}

While there has been a clear increase in the number of publications from selected Middle Eastern countries, there is need to improve the quality and reporting of studies. A standardised approach to quantifying medication errors' prevalence, severity, outcomes and contributory factors is warranted.

Acknowledgements Open Access funding provided by the Qatar National Library. The authors would like to acknowledge the contribution of Doua Al Saad to quality assessment.

Author contributions Binny Thomas reviews conception, protocol design, data collection, analysis, interpretation, drafting manuscript.

Vibhu Paudyal reviews conception, protocol design, data collection, analysis, interpretation, reviewing and approving final manuscript.

Katie MacLure reviews conception, protocol design, data collection, analysis, interpretation, reviewing and approving final manuscript.

Abdulrouf Pallivalapila: data collection, analysis, reviewing and approving final manuscript.

James McLay, reviews conception, protocol design, interpretation, reviewing and approving final manuscript.

Wessam El Kassem: data collection, analysis, reviewing and approving final manuscript.

Moza Al Hail: data collection, analysis, reviewing and approving final manuscript.

Derek Stewart reviews conception, protocol design, data collection, analysis, interpretation, reviewing and approving final manuscript.

All authors agree to be accountable for all aspects of the work in ensuring that questions related to the accuracy or integrity of any part of the work are appropriately investigated and resolved. 
Funding This systematic review was undertaken as part of the selffunded $\mathrm{PhD}$ at Robert Gordon University, UK.

Open Access This article is distributed under the terms of the Creative Commons Attribution 4.0 International License (http:// creativecommons.org/licenses/by/4.0/), which permits unrestricted use, distribution, and reproduction in any medium, provided you give appropriate credit to the original author(s) and the source, provide a link to the Creative Commons license, and indicate if changes were made.

\section{References}

1. Kohn LT, Corrigan JM, Donaldson MS (2000) To err is human: building a safer health system. National Academies Press

2. Jha AK, Larizgoitia I, Audera-Lopez C, Prasopa-Plaizier N, Waters H, Bates DW (2013) The global burden of unsafe medical care: analytic modelling of observational studies. BMJ Qual Saf 22: 809-815

3. World Health Organization. Medication Without Harm (2017) WHO global patient safety challenge. World Health Organization, Geneva

4. Donaldson LJ, Kelley ET, Dhingra-Kumar N, Kieny MP, Sheikh A (2017) Medication without harm: WHO's third global patient safety challenge. Lancet 389:1680-1681

5. Ghaleb MA, Barber N, Franklin BD, Yeung VW, Khaki ZF, Wong IC (2006) Systematic review of medication errors in pediatric patients. Ann Pharmacother 40(10):1766-1776

6. Miller MR, Robinson KA, Lubomski LH, Rinke ML, Pronovost PJ (2007) Medication errors in paediatric care: a systematic review of epidemiology and an evaluation of evidence supporting reduction strategy recommendations. BMJ Qual Saf 16(2):116-126

7. Lewis PJ, Dornan T, Taylor D, Tully MP, Wass V, Ashcroft DM (2009) Prevalence, incidence and nature of prescribing errors in hospital inpatients. Drug Saf 32(5):379-389

8. Ross S, Bond C, Rothnie H, Thomas S, Macleod MJ (2009) What is the scale of prescribing errors committed by junior doctors? A systematic review. Br J Clin Pharmacol 67:629-640

9. Tully MP, Ashcroft DM, Dornan T, Lewis PJ, Taylor D, Wass V (2009) The causes of and factors associated with prescribing errors in hospital inpatients. Drug Saf 32(10):819-836

10. Alsulami Z, Conroy S, Choonara I (2013) Medication errors in the Middle East countries: a systematic review of the literature. Eur J Clin Pharmacol 69:995-1008

11. Keers RN, Williams SD, Cooke J, Ashcroft DM (2013) Causes of medication administration errors in hospitals: a systematic review of quantitative and qualitative evidence. Drug Saf 36(11):1045-1067

12. Keers RN, Williams SD, Vattakatuchery JJ, Brown P, Miller J, Prescott L, Ashcroft DM (2014) Prevalence, nature and predictors of prescribing errors in mental health hospitals: a prospective multicentre study. BMJ Open 4(9):e006084

13. Metsälä E, Vaherkoski U (2014) Medication errors in elderly acute care-a systematic review. Scand J Caring Sci 28(1):12-28

14. Karthikeyan MB, Khaleel MI, Sahl M (2015) A systematic review on medication errors. IJDDR:7-4

15. Salmasi S, Khan TM, Hong YH, Ming LC, Wong TW (2015) Medication errors in the southeast Asian countries: a systematic review. PLoS One 10(9):e0136545

16. Alanazi MA, Tully MP, Lewis PJ (2016) A systematic review of the prevalence and incidence of prescribing errors with high-risk medicines in hospitals. J Clin Pharm Ther 41(3):239-245

17. Brown CL, Mulcaster HL, Triffitt KL, Sittig DF, Ash JS, Reygate K, Husband AK, Bates DW, Slight SP (2016) A systematic review of the types and causes of prescribing errors generated from using computerized provider order entry systems in primary and secondary care. J Am Med Inform:ocw119

18. Alshehri GH, Keers RN, Ashcroft DM (2017) Frequency and nature of medication errors and adverse drug events in mental health hospitals: a systematic review. Drug Saf 40(10):871-886

19. Salmasi S, Wimmer BC, Khan TM, Patel RP, Ming LC (2017) Quantitative exploration of medication errors among older people: a systematic review. Drugs Ther Perspect:1-9

20. Feinstein MM, Pannunzio AE, Castro P (2018) Frequency of medication error in pediatric anesthesia: a systematic review and metaanalytic estimate. Pediatr Anesth 28(12):1071-1077

21. Mekonnen AB, Alhawassi TM, McLachlan AJ, Brien JE (2018) Adverse drug events and medication errors in African hospitals: a systematic review. Drugs-real World Outcomes 5:1-24

22. Reason J (2000) Human error: models and management. BMJ (Clinical research ed) 320(7237):768-770

23. Shamseer L, Moher D, Clarke M, Ghersi D, Liberati A, Petticrew M, Shekelle P, Stewart LA, the PRISMA-P Group (2015) Preferred reporting items for systematic review and meta-analysis protocols (PRISMA-P) 2015: elaboration and explanation. BMJ 349:g7647

24. Thomas B, Pallivalapilla AR, Al Hail M, El Kassem W, Al Saad D, Singh R, et al (2015) Incidence, nature and causes of medication errors in hospitalised patients in Middle Eastern countries: a systematic review protocol. PROSPERO; CRD42015019693

25. Von Elm E, Altman DG, Egger M, Pocock SJ, Gøtzsche PC, Vandenbroucke JP et al (2007) The strengthening the reporting of observational studies in epidemiology (STROBE) statement: guidelines for reporting observational studies. PLoS Med 4:e296

26. National Coordinating Council for Medication Error Reporting and Prevention. Available at http://www.nccmerp.org [accessed December 2018]

27. Aronson JK (2009) Medication errors: definitions and classification. Brit J Clin Pharmacol 67(6):599-604

28. Anon (1993) ASHP guidelines on preventing medication errors in hospitals. Am J Hosp Pharm 50(2):305-314

29. Dean B, Barber N, Schachter M (2000) What is a prescribing error? BMJ Qual Saf 9(4):232-237

30. Aspden P, Wolcott JA, Bootman JL (2007) Cronenwett LR (2007) preventing medication errors. National Academies Press, Washington DC

31. Abdar ZE, Tajaddini H, Bazrafshan A, Khoshab H, Tavan A, Afsharpoor G, Amiri RNM, Rafiei H, Abdar ME (2014) Registered nurses perception of medication errors: a cross sectional study in Southeast of Iran. Int J Nurs Educ 6(1):19

32. Ali S, Aboheimed NI, Al-Zaagi I, Al-Dossari D (2017) Analysis of medication errors at a large tertiary care hospital in Saudi Arabia: a retrospective analysis. Int J Clin Pharm 39(5):1004-1007

33. Alshaikh M, Mayet A, Aljadhey H (2013) Medication error reporting in a university teaching hospital in Saudi Arabia. J Patient Saf 9(3):145-149

34. Al-Shara M (2011) Factors contributing to medication errors in Jordan: a nursing perspective. Iranian J N Midwifery Res 16(2): 158-161

35. Pawluk S, Jaam M, Hazi F, Al Hail MS, El Kassem W, Khalifa H et al (2017) A description of medication errors reported by pharmacists in a neonatal intensive care unit. Int J Cin Pharm 39(1):88-94

36. Al Tehewy M, Fahim H, Gad NI, El Gafary M, Rahman SA (2016) Medication administration errors in a university hospital. J Patient Saf 12(1):34-39

37. Bagheri-Nesami M, Esmaeili R, Tajari M (2015) Intravenous medication administration errors and their causes in cardiac critical care units in Iran. Materia Sociomedica 27(6):442-446

38. Cheragi MA, Manoocheri H, Mohammadnejad E, Ehsani SR (2013) Types and causes of medication errors from nurse's viewpoint. Iranian J N Midwifery Res 18(3):228-231 
39. Dibbi HM, Al-Abrashy HF, Hussain WA, Fatani MI, Karima TM (2006) Causes and outcome of medication errors in hospitalized patients. Saudi M J 27(10):1489-1492

40. Ehsani SR, Cheraghi MA, Nejati A, Salari A, Esmaeilpoor AH, Nejad EM (2013) Medication errors of nurses in the emergency department. J Med Ethics Hist Med 6:11

41. Farzi S, Irajpour A, Saghaei M, Ravaghi H (2017) Causes of medication errors in intensive care units from the perspective of healthcare professionals. J Res Pharm Pract 6(3):158-165

42. Fathi A, Hajizadeh M, Moradi K, Zandian H, Dezhkameh M, Kazemzadeh S, Rezaei S (2017) Medication errors among nurses in teaching hospitals in the west of Iran: what we need to know about prevalence, types, and barriers to reporting. Epidemiol Health 39:e2017022

43. Güneş ÜY, Gürlek Ö, Sönmez M (2014) Factors contributing to medication errors in Turkey: nurses' perspectives. J Nurs Manag 22(3):295-303

44. Hammoudi BM, Ismaile S, Abu Yahya O (2017) Factors associated with medication administration errors and why nurses fail to report them. Scand J Caring Sci 32(3):10381046

45. Mrayyan MT, Shishani K, Al-Faouri I (2007) Rate, causes and reporting of medication errors in Jordan: nurses' perspectives. J Nurs Man 15(6):659-670

46. Mrayyan MT (2012) Reported incidence, causes, and reporting of medication errors in teaching hospitals in Jordan: a comparative study. Contemp Nurse 41(2):216-232

47. Pazokian M, Zagheri Tafreshi M, Rassouli M (2014) Iranian nurses' perspectives on factors influencing medication errors. Int Nurs Rev 61(2):246-254

48. Shahrokhi A, Ebrahimpour F, Ghodousi A (2013) Factors effective on medication errors: a nursing view. J Res Pharm Pract 2(1):18-23

49. Shehata ZHA, Sabri NA, Elmelegy AA (2015) Descriptive analysis of medication errors reported to the Egyptian national online reporting system during six months. J Am Med Inform Assoc 23(2):366-374

50. SHoHani M, Tavan H (2018) Factors affecting medication errors from the perspective of nursing staff. J Clin Diagn Res 12(3)

51. Toruner EK, Uysal G (2012) Causes, reporting, and prevention of medication errors from a pediatric nurse perspective. Aust J Adv Nurs 29(4):28

52. Vazin A, Delfani S (2012) Medication errors in an internal intensive care unit of a large teaching hospital: a direct observation study. Acta Med Iran 50(6):425-432

53. Al-Yousiff SAA, Mohamed N (2013) Nurses' experiences toward perception of medication administration errors reporting. IOSR J Nurs Health Scienc 1(4):56-70

54. Gorgich EA, Barfroshan S, Ghoreishi G, Yaghoobi M (2016) Investigating the causes of medication errors and strategies to prevention of them from nurses and nursing student viewpoint. Glob J Health Sci 8(8):54448

55. Moher D, Liberati A, Tetzlaff J, Altman DG (2009) Preferred reporting items for systematic reviews and metaanalyses: the PRISMA statement. Ann Intern Med 151(4): 264-269

56. EQUATOR Network, Enhancing the quality and transparency of health research Available at http://www.equator-network.org/ [Accessed December 2018]

57. Garfield S, Reynolds M, Dermont L, Franklin BD (2013) Measuring the severity of prescribing errors: a systematic review. Drug Saf 36(12):1151-1157

58. Craig P, Dieppe P, Macintyre S, Michie S, Nazareth I, Petticrew M (2008) Developing and evaluating complex interventions: the new Medical Research Council guidance. BMJ 337:a1655

59. Abbasinazari M, Talasaz AH, Mousavi Z, Zare-Toranposhti S (2013) Evaluating the frequency of errors in preparation and administration of intravenous medications in orthopedic, general surgery and gastroenterology wards of a teaching hospital in Tehran. IJPR 12(1):229-234

60. Abbasinazari M, Talasaz AH, Eshraghi A, Sahraei Z (2013) Detection and management of medication errors in internal wards of a teaching hospital by clinical pharmacists. Acta Med Iran 51(7): 482-486

61. Ahmed SE, Al-Abbas SA, Al-Omran AS, Sadat-Ali M, Al-Shafei BA, Al-Turki RA (2010) Medication administration errors in Eastern Saudi Arabia. Saudi Med J 31(11):1257-1259

62. Alakahli KM, Alzomar AK, Mohammad AAS (2014) Evaluation of medication error in intensive care unit in Yemeni hospitals. J Appl Pharm 6(3):247-251

63. Al-Dhawailie A (2011) Inpatient prescribing errors and pharmacist intervention at a teaching hospital in Saudi Arabia. Saudi Pharm J 19(3):193-196

64. Al-Hajje A, Awada S, Rachidi S (2012) Medication prescribing errors: data from seven Lebanese hospitals. Liban Leban Med J 60(1):37-44

65. Ali MAS, Lobos CM, Abdelmegid MAF, El-Sayed A (2017) The frequency and nature of medication errors in hospitalized patients with acute coronary syndrome. Int $\mathrm{J}$ Clin Pharm 39(3):542-550

66. Al-Jeraisy MI, Alanazi MQ, Abolfotouh MA (2011) Medication prescribing errors in a pediatric inpatient tertiary care setting in Saudi Arabia. BMC Res Notes 4(1):294

67. Aljadhey H, Mahmoud MA, Mayet A, Alshaikh M, Ahmed Y, Murray MD et al (2013) Incidence of adverse drug events in an academic hospital: a prospective cohort study. IJQHC 25(6):648655

68. Al-Ramahi R, Hmedat B, Alnjajrah E, Manasrah I, Radwan I, Alkhatib M (2017) Medication dosing errors and associated factors in hospitalized pediatric patients from the South Area of the West Bank - Palestine. Saudi Pharm J 25(6): $857-860$

69. Arabi Y, Alamry A, Al Owais SM, Al-Dorzi H, Noushad S, Taher S (2012) Incident reporting at a tertiary care hospital in Saudi Arabia. J Patient Saf 8(2):81-87

70. Dabaghzadeh F, Rashidian A, Torkamandi H, Alahyari S, Hanafi S, Farsaei S, Javadi M (2013) Medication errors in an emergency department in a large teaching hospital in Tehran. IJPR 12(4): 937-942

71. El-Shazly AN, Al-Azzouny MA, Soliman DR, Abed NT, Attia SS (2017) Medical errors in neonatal intensive care unit at Benha University Hospital, Egypt. Eastern Mediterr Health J 23(1):31-39

72. Fahimi F, Ariapanah P, Faizi M, Shafaghi B, Namdar R, Ardakani MT (2008) Errors in preparation and administration of intravenous medications in the intensive care unit of a teaching hospital: an observational study. Aust Crit Care 21(2):110-116

73. Fahimi F, Nazari MA, Abrishami R, Sistanizad M, Mazidi T, Faghihi T et al (2009) Transcription errors observed in a teaching hospital. Arch Iran Med 12(2)

74. Fahimi F, Sefidani Forough A, Taghikhani S, Saliminejad L (2015) The rate of physicochemical incompatibilities, administration errors. Factors correlating with nurses' errors. IJPR 14(Suppl:87-93

75. Gharekhani A, Kanani N, Khalili H, Dashti-Khavidaki S (2014) Frequency, types, and direct related costs of medication errors in an academic nephrology ward in Iran. Ren Fail 36(8):1268-1272

76. Hamishehkar H, Khamechi M, Naghili B, Rezaee H (2014) Evaluation of medication errors in an infectious diseases ward of a teaching hospital in Iran. Pharm Sci 20(2):66

77. Hammour KA, Jalil MHA (2016) Medication errors in voluntary reported incidents at a Jordanian hospital. J Med J 50(2):87-96

78. Kandil M, Sayyed T, Emarh M, Ellakwa H, Masood A (2012) Medication errors in the obstetrics emergency ward in a low resource setting. J Matern-Fetal Neonatal Med 25(8):1379-1382 
79. Khammarnia M, Sharifian R, Keshtkaran A, Zand F, Barati O, Khonia N, Setoodehzadeh F (2015) Prescribing errors in two ICU wards in a large teaching hospital in Iran. Int J Risk Saf Med 27(4): $169-175$

80. Lustig A (2000) Medication error prevention by pharmacists-an Israeli solution. PWS 22(1):21-25

81. Saravi BM, Mardanshahi A, Ranjbar M, Siamian H, Azar MS, Asghari Z, Motamed N (2015) Rate of medical errors in affiliated hospitals of Mazandaran University of Medical Sciences. Mater Soc 27(1):31-34

82. Sulaiman ZH, Hamadi SA, Obeidat NM, Basheti IA (2017) Evaluating medication errors for hospitalized patients: the Jordanian experience. JJPS 10(2):87-101

83. Vessal G (2010) Detection of prescription errors by a unit-based clinical pharmacist in a nephrology ward. PWS 32(1):59-65
84. Zeraatchi A, Talebian MT, Nejati A, Dashti-Khavidaki S (2013) Frequency and types of the medication errors in an academic emergency department in Iran: the emergent need for clinical pharmacy services in emergency departments. J Res Pharm Pract 2(3):118-122

Publisher's note Springer Nature remains neutral with regard to jurisdictional claims in published maps and institutional affiliations. 